\title{
OPTIMAL DISTRIBUTED FLOW CONTROL OVER MULTIPLE PARALLEL WIRELESS CHANNELS
}

\author{
Antoni Morell, José López Vicario and Gonzalo Seco-Granados \\ Universitat Autònoma de Barcelona (UAB) \\ Telecommunications and System Engineering Department (TES) \\ e-mail: \{Antoni.Morell,Jose.Vicario,Gonzalo.Seco\}@uab.cat
}

\begin{abstract}
This paper addresses a distributed flow and channel allocation method for Point-to-MultiPoint (PMP) networks operating over multiple parallel channels, where a central node shares its rate capacity with multiple subscribers. The problem formulation takes into account fairness and Quality of Service (QoS) considerations by means of generalized utility functions. Assuming that a local area network (LAN), wired or wireless, is attached to each of the subscribers, a centralized allocation scheme becomes prohibitive in terms of required network signalling when the number of connections within the LANs become high. In order to overcome this and to keep practicality in terms of iterations needed to converge, we apply a combination of a Mean Value Cross (MVC) decomposition strategy with the novel coupled-decompositions method that achieves the optimal solution with a reduced number of iterations when compared to classical approaches.
\end{abstract}

\section{INTRODUCTION}

The immediate future of wireless communications and networks is probably related to the popularization of novel services such as video streaming, voice over IP, online gaming or new internet applications (e.g. social networks). In order to absorb the forthcoming capacity increase, adequate Radio Resource Management (RRM) procedures come into the equation. Moreover, the last decade has shown us the benefits of jointly optimizing multiple system layers (i.e. the so called cross-layer designs) and a recent research line is focused on establishing the optimal interactions across system layers and also across the elements in a network [1] by means of employing decomposition techniques [2, Sec. 6.4], [3].

This paper explores practical algorithms to perform flow control jointly with channel allocation when a backbone wireless network with parallel channels is the bottleneck for the ongoing connections. In particular, the coupled-decompositions method derived by the authors in [4] (particular case) and [3, Sec. 3.3] (general case) has been applied.

This work was supported in part by the Spanish Ministry of Science and Innovation under TEC2008-06305 project.

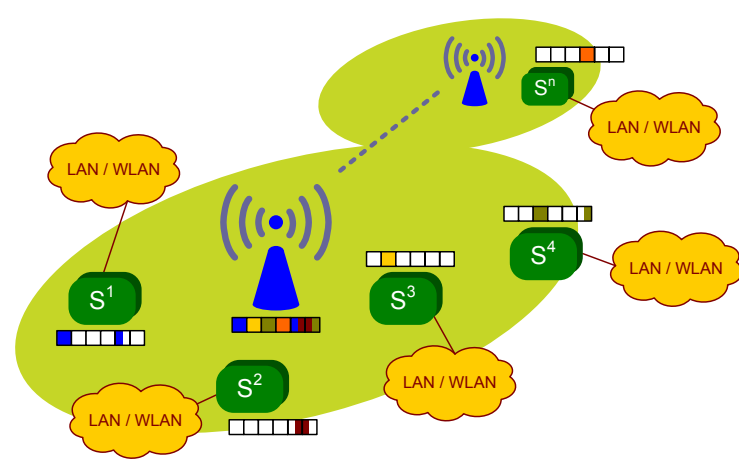

Fig. 1. Network reference model.

The main difference between our solution and existing contributions $[5,6]$ is that our approach is more practical in terms of required signalling and iterations needed to converge and furthermore, it does not sacrifice optimality. Those drawbacks detected in existing techniques have been improved at the expenses of focusing on a more specialized design (i.e. a point-to-multipoint scenario). Note that in wireless networks the ever-changing channel condition requires higher adaptation capacity. This is achieved with our scheme.

\section{SYSTEM MODEL}

Let us consider a wireless network with a Point-to-MultiPoint (PMP) topology as depicted in Figure 1. A central node (CN) with a fixed transport capacity $C$ serves a number of subscribers, which are in charge of managing the multiple connections within the local area networks (LANs), wired or wireless, that are below them. Without loss of generality, a LAN can also be substituted by a single end node driving one or multiple services. We assume that there are no bottlenecks inside the local networks and thus, the following approach is also valid in case a subscriber is attached to the central node through a transparent repeater that does not limit the transport rate between the subscriber and the base station, as it is shown in the right top corner of Figure 1.

Each subscriber is able to establish a transmission using one or multiple channels and each channel can be further sub- 
divided by simple time sharing among the subscribers. We further assume that the transmit power is fixed and that each user experiences a different slow fading gain in each channel. Let us group the corresponding achievable rates of the i-th subscriber in the vector $\boldsymbol{c}_{i}=\left[c_{i, 1}, \ldots, c_{i, N_{c}}\right]^{T}$, where $N_{c}$ is the total number of channels. Similarly, the variables in $\boldsymbol{\rho}_{i}=\left[\rho_{i, 1}, \ldots, \rho_{i, N_{c}}\right]^{T}$ represent the channels allocation to the $\mathrm{i}$-th subscriber and take values $0 \leq \rho_{i, k} \leq 1$ (i.e., timesharing of a given channel among users is allowed).

Given the problem constraints, the objective is to establish a criterion guiding both the flow control and the radio resource allocation. The former decides the transmission rate of each connection within the network whereas the latter is devoted to the allocation of radio resources (channels in our case) to the subscribers. An accepted design criterion is to keep fairness among users and it can be achieved by means of maximizing the sum of the utilities of all flows in a network, i.e. the so-called Network Utility Maximization (NUM) approach. As discussed in [7], a series of concave utility functions are defined as

$$
U_{j}\left(r_{j} ; p_{j}, \alpha\right)=\left\{\begin{array}{cc}
p_{j} \log \left(r_{j}\right), & \alpha=1 \\
p_{j} \frac{r_{j}^{(1-\alpha)}}{1-\alpha}, & \alpha \neq 1
\end{array},\right.
$$

where $r_{j}$ is the rate of the $\mathrm{j}$-th flow in the network and $p_{j}$ is a parameter used to prioritize $r_{j}$ against the other rates. Finally, $\alpha$ is common to all flows and fixes the degree of fairness in the network [7]. Using this, our problem is represented in convex form as

$$
\begin{aligned}
& \text { JOINT FLOW CONTROL \& CHANNEL ALLOCATION: } \\
& \begin{array}{ccc}
\max _{\left\{r_{j}^{i}\right\}, \Gamma} & \sum_{i=1}^{N} \sum_{j=1}^{N_{i}} U_{j}^{i}\left(r_{j}^{i} ; p_{j}^{i}, \alpha\right) & \\
\text { s.t. } & \sum_{i=1}^{N} \sum_{j=1}^{N_{i}} r_{j}^{i} \leq C & \\
& \sum_{j=1}^{N_{i}} r_{j}^{i} \leq c^{i}\left(\boldsymbol{\rho}_{i}\right), & i=1, \ldots, N, \\
& m_{j}^{i} \leq r_{j}^{i} \leq d_{j}^{i}, & \forall i, \forall j \\
\Gamma \mathbf{1} \preceq \mathbf{1} & \\
\boldsymbol{\rho}_{i} \succeq \mathbf{0}, & i=1, \ldots, N
\end{array}
\end{aligned}
$$

where $m_{j}^{i}$ and $d_{j}^{i}$ are the minimum required and the maximum allowed values for the rates. The minimum values are generally associated with QoS requirements. The maximums can represent requests in Demand Assignment Multiple Access approaches or they can be obviated otherwise. Finally, $\Gamma=\left[\boldsymbol{\rho}_{1}, \ldots, \boldsymbol{\rho}_{N}\right]$ is a matrix that collects the allocation vectors of the $N$ subscribers, $N_{i}$ is the number of ongoing connections managed by the i-th subscriber, $c^{i}\left(\boldsymbol{\rho}_{i}\right)=\boldsymbol{\rho}_{i}^{T} \boldsymbol{c}_{i}, \mathbf{1}$ is an all-ones column vector and $\succeq \mid \preceq$ stand for componentwise inequalities.

\section{CONVEX DECOMPOSITION-BASED SOLUTION}

Even given that it is possible to solve (2) centrally using standard convex optimization methods (e.g. interior point methods [8, Ch. 11]), we want to alleviate the signalling needs of this approach (i.e. transmission of connection descriptors $m_{j}^{i}, d_{j}^{i}$ and $p_{j}^{i}$ ) by means of distributing the optimization process thanks to decomposition strategies. Classical schemes such as primal and dual decomposition [2, Sec. 6.4] fail in terms of convergence speed and have been discarded. Instead, the Mean Value Cross (MVC) decompositions method [9] has been used to decouple the original problem into one channel allocation problem and one flow allow allocation problem. The former can be solved centrally at the base station since all the information is available and the latter applies the novel coupled-decompositions method $[4,3]$ in order to achieve the optimal rate allocation in a few iterations.

\subsection{Separation into Flow Control and Channel Alloca- tion}

Let us rewrite (2) as

$$
\begin{array}{cc}
\max _{\left\{r_{j}^{i}\right\}, \Gamma} & \sum_{i=1}^{N} \sum_{j=1}^{N_{i}} U_{j}^{i}\left(r_{j}^{i} ; p_{j}^{i}, \alpha\right) \\
\text { s.t. } & \sum_{j=1}^{N_{i}} r_{j}^{i} \leq \boldsymbol{\rho}_{i}^{T} \boldsymbol{c}_{i}, \quad i=1, \ldots, N \\
& \left\{r_{j}^{i}\right\} \in \mathcal{R} \\
& \left\{\boldsymbol{\rho}_{i}\right\} \in \mathcal{S}
\end{array}
$$

where $\mathcal{R}=\left\{r_{j}^{i} \mid m_{j}^{i} \leq r_{j}^{i} \leq d_{j}^{i}, \sum_{i, j} r_{j}^{i} \leq C\right\}$ and $\mathcal{S}=$ $\left\{\boldsymbol{\rho}_{i} \mid \Gamma \mathbf{1} \preceq \mathbf{1}, \boldsymbol{\rho}_{i} \succeq \mathbf{0}\right\}$. The MVC decompositions method forms a partial Lagrangian that relaxes a subset of the constraints in the problem and from this Lagrangian, it obtains one primal/dual subproblem by fixing the primal/dual variables. It is shown in [9] that adequately combining both subproblems, as will be described later, leads to the optimal solution. In our case, the constraints $\sum_{j=1}^{N_{i}} r_{j}^{i} \leq \boldsymbol{\rho}_{i}^{T} \boldsymbol{c}_{i}$ are relaxed using dual variables $\gamma_{i}$. The resulting primal subproblem is

FLOW CONTROL :

$$
\begin{array}{cc}
\max _{\left\{r_{j}^{i}\right\}} & \sum_{i=1}^{N} \sum_{j=1}^{N_{i}} U_{j}^{i}\left(r_{j}^{i} ; p_{j}^{i}, \alpha\right) \\
\text { s.t. } & \sum_{\substack{N_{i} \\
N_{j}}}^{r_{j}^{i} \leq \boldsymbol{\rho}_{i}^{T} \boldsymbol{c}_{i}, \quad} \quad i=1, \ldots, N \\
& \left\{r_{j}^{i}\right\} \in \mathcal{R}
\end{array}
$$

for fixed $\left\{\boldsymbol{\rho}_{i}\right\}$ values. The dual subproblem is

$$
\begin{array}{cl}
\max _{\left\{r_{j}^{i}\right\},\left\{\boldsymbol{\rho}_{i}\right\}} & \sum_{i} \sum_{j} U_{j}^{i}\left(r_{j}^{i} ; p_{j}^{i}, \alpha\right)-\gamma_{i}\left(\sum_{j=1}^{N_{i}} r_{j}^{i}-\boldsymbol{\rho}_{i}^{T} \boldsymbol{c}_{i}\right) \\
\text { s.t. } & \left\{r_{j}^{i}\right\} \in \mathcal{R} \\
& \left\{\boldsymbol{\rho}_{i}\right\} \in \mathcal{S}
\end{array}
$$

for fixed $\left\{\gamma_{i}\right\}$ values. Note that the maximization in (5) can be done independently in $\left\{\boldsymbol{\rho}_{i}\right\}$ and $\left\{r_{j}^{i}\right\}$ and thus, the channel allocation variables are updated by solving the linear program

CHANNEL ALLOCATION :

$$
\begin{array}{cc}
\max _{\left\{\boldsymbol{\rho}_{i}\right\}} & \sum_{i=1}^{N} \gamma_{i} \cdot\left(\boldsymbol{\rho}_{i}^{T} \boldsymbol{c}_{i}\right) \\
\text { s.t. } & \left\{\boldsymbol{\rho}_{i}\right\} \in \mathcal{S}
\end{array},
$$

which can be centrally computed. Observe that our strategy based in MVC decomposition is different to other pure dual decomposition-based approaches in the literature, e.g. [10]. 


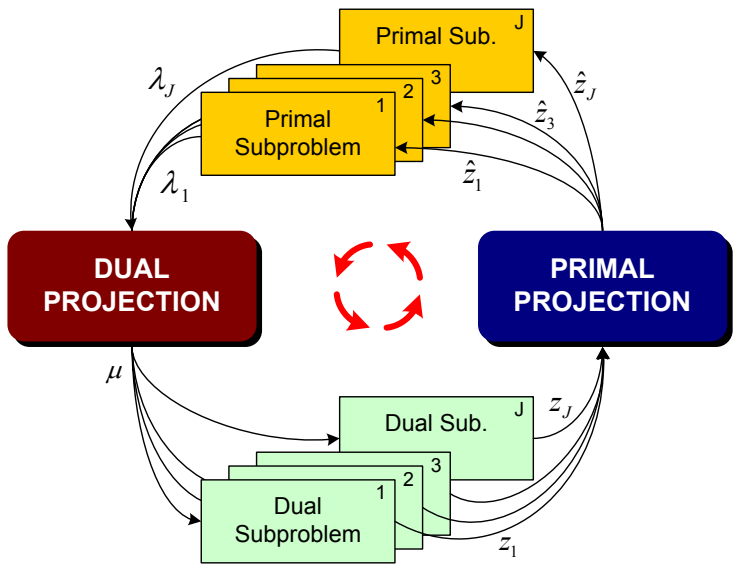

Fig. 2. Coupled-decompositions method.

The joint problem is then solved applying the MVC decompositions method as follows:

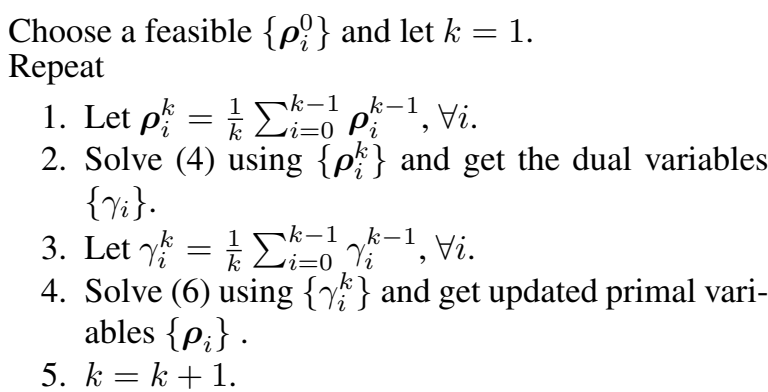

Until convergence.

\subsection{Flow Control}

The flow control problem in step 2 still needs to gather a huge amount of control data from the other nodes if computed at the base station. In order to avoid this situation, we apply the novel coupled-decompositions method to (4). In the following, a very short review of the method is provided.

Let us consider the following general formulation,

$$
\begin{array}{ccc}
\max _{\left\{\boldsymbol{x}_{j}\right\}, \boldsymbol{z}} & \sum_{j=1}^{J} f_{j}\left(\boldsymbol{x}_{j}\right) & \\
\text { s.t. } & \boldsymbol{x}_{j} \in \mathcal{X}_{j}, & j=1, \ldots, J \\
& h_{j}\left(\boldsymbol{x}_{j}\right) \leq z_{j}, & j=1, \ldots, J \\
& \sum_{j=1}^{J} z_{j} \leq B & \\
& \boldsymbol{z} \in \mathcal{Z}, & \mathcal{Z}=\mathcal{Z}_{1} \times \ldots \times \mathcal{Z}_{J}
\end{array}
$$

where $\left\{f_{j}\left(\boldsymbol{x}_{j}\right), h_{j}\left(\boldsymbol{x}_{j}\right)\right\}$ are convex functions and the subsets $\left\{\mathcal{X}_{j}, \mathcal{Z}_{j}\right\}$ are also convex ( $\mathcal{Z}_{j}$ is one-dimensional). The algorithm used to numerically solve (7) is summarised in Figure 2. First of all, note that (7) suits the classic decompositions [2, Sec 6.4] so that it is possible to define a primal (dual) master problem that coordinates $J$ independent primal (dual) subproblems (and the idea differs from the MVC principle). Given this, the key point in coupled-decompositions is to provide the mechanism to intertwine both approaches in a single algorithm, which requires the redefinition of the master problems into the projections. The subproblems are identical.

The algorithm starts with an initial value of the Lagrange multiplier $\mu$ associated with the constraint $\sum_{i=1}^{J} z_{i} \leq B$, which is used by the dual subproblems to obtain the $z$ candidates. If these $z$ candidates exceed the problem constraints, the primal projection corrects the situation. Afterwards, the adjusted values $\hat{z}$ are used by the primal subproblems to obtain the $\lambda_{i}$ candidates to the dual variable $\mu$ and finally, the dual projection updates $\mu$ by choosing the adequate candidate among $\left\{\lambda_{i}\right\}$ that guarantees the convergence of the method. The interested reader can find a more detailed description of the technique as well as a formal proof in [3, Sec. 3.3.6].

In the sequel, we detail the proposed strategy to solve our specific flow control problem. First, let us define $\boldsymbol{y}=$ $\left[y^{1}, \ldots, y^{N}\right]^{T}$ and rewrite (4) as

$$
\begin{array}{ll}
\max _{\left\{y^{i}\right\}} & \sum_{i=1}^{N} U^{i}\left(y^{i}\right) \\
\text { s.t. } & \sum_{\substack{i=1 \\
\boldsymbol{y}}}^{N} y^{i} \leq C \\
& \boldsymbol{y},
\end{array}
$$

where $\mathcal{Y}=\left\{y^{i} \mid y^{i} \leq \boldsymbol{\rho}_{i}^{T} \boldsymbol{c}_{i}, M^{i} \preceq y^{i} \preceq D^{i}\right\}$. The values $M^{i}$ and $D^{i}$ are $M^{i}=\sum_{j=1}^{N_{i}} m_{j}^{i}$ and $D^{i}=\sum_{j=1}^{N_{i}} d_{j}^{i}$, respectively. The definition of $U^{i}\left(y^{i}\right)$ follows:

$$
U^{i}\left(y^{i}\right)=\left\{\begin{array}{cc}
\max _{\left\{r_{j}^{i}\right\}} & \sum_{j=1}^{N_{i}} U_{j}^{i}\left(r_{j}^{i} ; p_{j}^{i}, \alpha\right) \\
\text { s.t. } & \sum_{j=1}^{N_{i}} r_{j}^{i} \leq y^{i} \\
& m_{j}^{i} \leq r_{j}^{i} \leq d_{j}^{i}
\end{array} .\right.
$$

Note that both (8) and (9) can be cast into the formulation of (7) if functions, variables and subsets are adequately chosen. Furthermore, given that problems (8) and (9) are nested, the following steps of a 2-layer coupled-decompositions approach (see Figure 3) achieve a distributed flow control,

Choose an initial $\mu^{0}$.

Repeat

1. The dual variable at time instant $t, \mu^{t}$ (associated with $\sum_{i=1}^{N} y^{i} \leq C$ ), is sent to the subscribers and reaches each connection through the LANs.

2. Each ongoing connection computes its flow allocation given $\mu^{t}$ by means of solving the inner dual subproblems. Thereafter, the subscribers as well as the $\mathrm{CN}$ compute their aggregated bit rates.

3. The $\mathrm{CN}$ corrects the previous allocations (primal projection) in order to attain $\sum_{i=1}^{N} y^{i} \leq C, \boldsymbol{y} \in$ $\mathcal{Y}$ and $y^{i} \leq \boldsymbol{\rho}_{i}^{T} \boldsymbol{c}_{i}, i=1, \ldots, N$.

4. The adjusted allocations allow the subscribers to obtain new $\gamma_{i}$ candidates by running the method again inside each local network.

5. Finally, the $\mathrm{CN}$ updates the value of the dual variable to $\mu^{t+1}$ using a subset of the new $\gamma_{i}$ values in the dual projection, namely $\left\{\lambda_{i}^{\prime}\right\}[3]$.

Until convergence. 

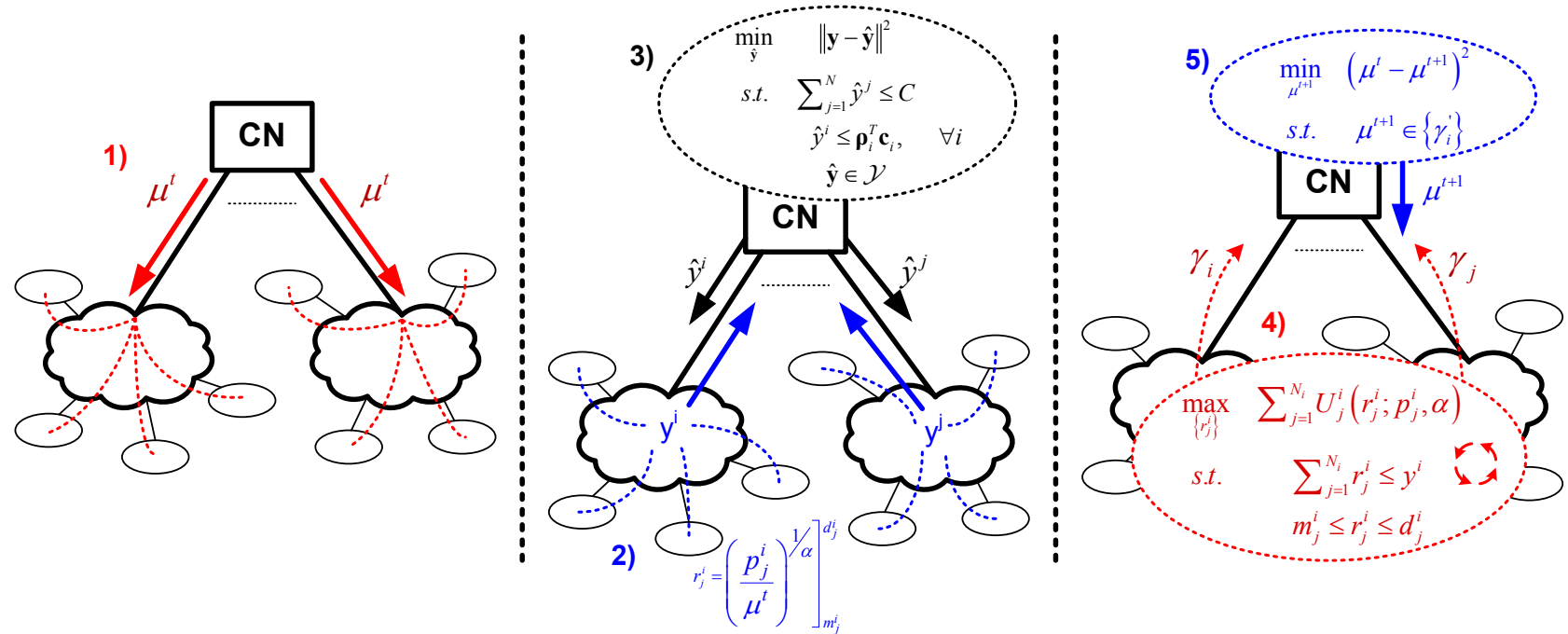

Fig. 3. Flow allocation using a 2-level coupled-decompositions approach.

Note in Figure 3 that only step 4 in the previous list performs inner iterations whereas a formal application of the method would require to iterate also in step 2. Notwithstanding, we have theoretically checked that the closed-form solution shown in Figure 3 suffices to guarantee the convergence to the optimal flow allocation. Intuitively, this multi-layer coupled-decompositions strategy tries to find a consensus on the price $\mu$ that has to be paid for sharing the transport capacity $C$ of the central node. All services in the network participate in obtaining such optimal value. However, the subscriber price $\gamma_{i}$ may differ from the global price $\mu$ if, for example, its link capacity is small (hence forcing the price to locally increase). In that situations, the channel allocation part must balance radio resources properly in order to reach a global fair solution. Next section shows some numerical results to exemplify this.

\section{NUMERICAL RESULTS}

Let us consider a a network with 3 subscribers managing 70 connections in total (20 attached to $S^{1}, 30$ to $S^{2}$ and 20 more to $S^{3}$ ) and 7 available channels. All ongoing connections have $m_{j}^{i}=1 \mathrm{kbps}, p_{j}^{1}=1$ and $\alpha=1$ (proportional fairness). Requested rates are $10 \mathrm{kbps}$ for connections within $S^{1}$, $40 \mathrm{kbps}$ for those through $S^{2}$ and finally $100 \mathrm{kbps}$ for connections managed by $S^{3}$. We further assume the following capacities per channel and subscriber (in kbps),

$$
\begin{gathered}
\boldsymbol{c}_{1}=[55,350,50,120,40,250,70]^{T}, \\
\boldsymbol{c}_{2}=[300,420,270,340,50,25,87]^{T}, \\
\boldsymbol{c}_{3}=[20,80,71,33,270,150,420]^{T},
\end{gathered}
$$

and that the $C=1200 \mathrm{kbps}$. Initially, $S^{1}$ uses the first channel, $S^{2}$ the second and $S^{3}$ the third so that the initial transport capacities are $55 \mathrm{kbps}, 420 \mathrm{kbps}$ and $71 \mathrm{kbps}$, respectively.
Once the proposed flow and channel allocation scheme has been executed, all the ongoing connections within $S^{1}$ reach their requests, i.e. 10kbps, whereas all the connections within $S^{2}$ and $S^{3}$ get 20kbps. Regarding channel allocation, the following result is obtained,

$$
\begin{gathered}
\boldsymbol{\rho}_{1}=[0,0.33,0,0,0,0.33,0]^{T}, \\
\boldsymbol{\rho}_{2}=[1,0.67,1,1,0,0,0]^{T}, \\
\boldsymbol{\rho}_{3}=[0,0,0,0,1,0.67,1]^{T},
\end{gathered}
$$

so that the final subscriber rates are $200 \mathrm{kbps}, 1191.4 \mathrm{kbps}$ and $790.5 \mathrm{kbps}$, respectively. As done in other works in the literature [6], we have used instantaneous values of $\left\{\gamma_{i}\right\}$ instead of their averaged version in order to improve the convergence properties (in practice these values are high in the first iterations and slow down the convergence of the algorithm if averaged). Figure 4 shows how initial channel allocation variables converge to the values in (11). Note that since the connections that go through $S^{1}$ (1-20) reach exactly their request, a total rate of $200 \mathrm{kbps}$ is required. On the contrary, connections $21-70$ are limited by $C=1200 \mathrm{kbps}$ and rates are equally distributed (20kbps each) as expected in a fair solution. In case $C \rightarrow \infty$, note that they would get $1191.4 k b p s / 30$ connec. $\approx$ $790.5 \mathrm{kbps} / 20 \mathrm{connec} . \approx 40 \mathrm{kbps} /$ connec. with the current channel allocation.

From a practical point of view, we are interested in systems that converge fast to the optimal solution. For that purpose, channel allocation can be truncated if necessary (e.g. around 50 iterations) in order to attain the required computational time without much performance loss. Notwithstanding, convergence properties of the flow allocation subpart of the method are more important since it must be executed at each allocation update. Furthermore, if we assume that subscriber achievable rates $\boldsymbol{\rho}_{i}^{T} \boldsymbol{c}_{i}$ as well as aggregated flows keep more or less constant during a period of time, it is only the flow control part of the method that runs. 


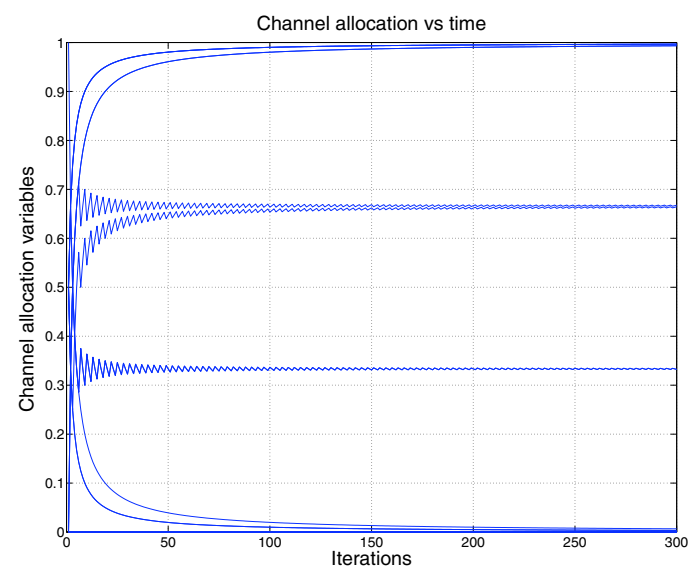

Fig. 4. Channel allocation variables versus time.

Figure 5 provides a performance comparison between the proposed 2-level coupled-decompostions method and a classical 2-level dual decomposition approach. Results show the evolution of the dual variable $\mu$ at the highest level (associated with $\sum_{i=1}^{N} y^{i} \leq C$ ) for both techniques. Whereas the proposed method finds the optimal value in less than 10 iterations, a dual decomposition strategy requires more than 500 iterations. Furthermore, the problem of choosing the adequate step-size in primal/dual decomposition (both methods follow a projected subgradient approach) is obviated. Finally, note the signalling reduction with respect to a centralized approach. In our scheme each subscriber exchanges only a primal variable (either $y^{i}$ or $\hat{y}^{i}$ ) and a dual variable (either $\mu$ or $\gamma_{i}$ ) with the base station and only for a few iterations.

\section{CONCLUSIONS}

This paper tackles the joint flow and channel allocation problem for point-to-multipoint networks that operate over multiple parallel channels (as in OFDM) where a central node, e.g. the base station, has to distribute a certain amount of transport rate among multiple subscribers. We assume that the available rate at each subscriber is locally distributed among many connections with different QoS requirements that form local area networks, either wireless or wired, and that impose no further link constraints.

In order to alleviate the stringent signalling requirements of a centralized approach (which grow with the number of ongoing connections within the local area networks), we have proposed a fair distributed decomposition technique that splits the original problem into a channel allocation plus a flow control mechanism. For the sake of practicality (i.e. low number of iterations) we have used a Mean Value Cross decompositions approach in the former and the novel coupleddecompoistions strategy in the latter. As a result, the number of iterations has been kept small when compared to classical designs, specially in the flow allocation part, which reduces
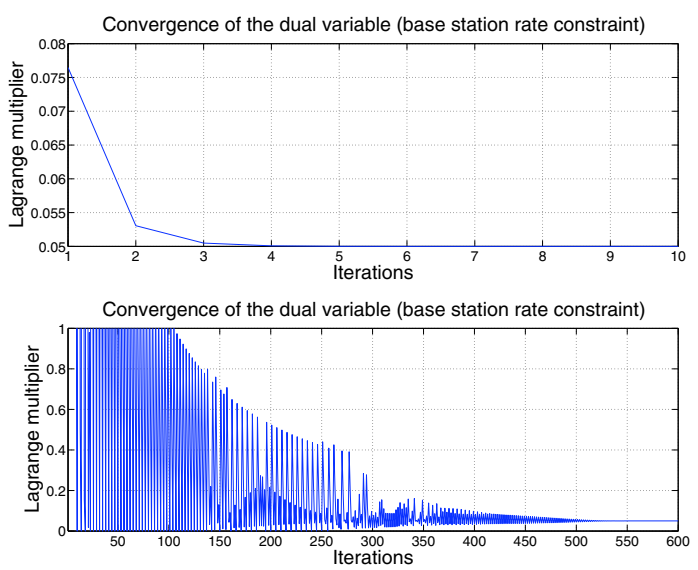

Fig. 5. Flow allocation computation: coupled-decompositions (top) versus dual decomposition (bottom).

the iteration count in two orders of magnitude with respect to known techniques. Furthermore, it can be stand-alone executed when the required updating period for the channel allocation is large.

\section{REFERENCES}

[1] M. Chiang, S.H. Low, A.R. Calderbank, and J.C. Doyle, "Layering as Optimization Decomposition: A Mathematical Theory of Network Architectures," Proceedings of the IEEE, Vol. 95, No. 1, pp. 255-312, Jan 2007.

[2] D.P. Bertsekas, Nonlinear Programming, Belmont, MA, USA: Athena Scientific, 1999.

[3] A. Morell, A Convex Decomposition Perspective on Dynamic Bandwidth Allocation and Applications, Ph.D. Dissertation available at http://spcomnav.uab.es, 2008.

[4] A. Morell, G. Seco-Granados, and M.A. Vázquez-Castro, "Efficient Cross-Layer Algorithm for Fair Dynamic Bandwidth Allocation," in proc. IEEE ICCCN, Honolulu, Aug 2007.

[5] L.B. Le and E. Hossain, "Joint Rate Control and Resource Allocation in OFDMA Wireless Mesh Networks," Proceedings IEEE WCNC, pp. 3041-3045, Mar 2007.

[6] P. Soldati, B. Johansson, and M. Johansson, "Distributed Optimization of End-to-End Rates and Radio Resources in WiMax Single-Carrier Networks," in proc. IEEE GLOBECOM, San Francisco (USA), Nov 2006.

[7] J. Mo and J. Walrand, "Fair End-to-end Window-based Congestion Control," IEEE/ACM Trans. Networking, Vol. 8, No. 5, pp. 556-567, Oct 2000.

[8] L. Boyd and S. Vandenberghe, Convex optimization, Cambridge University Press, 2003.

[9] K. Holmberg and K.C. Kiwiel, "Mean Value Cross Decomposition for Nonlinear Convex Problems," Optimization Methods and Software, Vol. 21, No. 3, pp. 401-417, Jun 2006.

[10] L. Xiao, M. Johansson, and S.P. Boyd, "Simultaneous routing and resource allocation via dual decomposition," IEEE Trans. on Communications, Vol. 52, No.7, pp. 1136-1144, Jul 2004. 